\title{
A implantação do balanced scorecard em empresas brasileiras sob a perspectiva da cultura nacional
}

\author{
Frederico Pires Pereira Fernandes \\ Professor da Universidade Federal do Rio de Janeiro - UFRJ \\ E-mail: fredpires@predialnet.com.br \\ Ana Carolina Pimentel Duarte da Fonseca \\ Professora da Universidade Federal do Rio de Janeiro - UFRJ \\ E-mail: anafonseca@pobox.com
}

\section{RESUMO}

Diversos autores, tais como Hofstede (1991), Hampden-Turner e Trompenaars (1995), e Caldas e Wood (1998), alertam que práticas gerenciais geralmente carregam características da cultura nacional daqueles que as desenvolveram. O Balanced Scorecard, modelo gerencial desenvolvido pelos pesquisadores americanos Kaplan e Norton, vem sendo adotado em diversos países fora dos Estados Unidos, inclusive no Brasil. A análise das dimensões culturais relevantes para a implantação do Balanced Scorecard resultou em algumas proposições teóricas acerca de sua implantação em empresas brasileiras, mostrando que os valores culturais implícitos no Balanced Scorecard parecem, em sua maioria, coincidir com os da cultura americana e divergir dos da cultura brasileira Os resultados da análise de cinco estudos de casos sobre a implantação do Balanced Scorecard em empresas brasileiras apontam para a influência das diferenças culturais durante o processo de implantação.

Palavras-chave: Balanced scorecard. Cultura organizacional. Cultura Nacional.

The implementation of Balanced Scorecard im brazilian companies under the national culture perspective

\begin{abstract}
According to considerations presented by Hofstede (1991), Hampden-Turner and Trompenaars (1995), and Caldas and Wood (1998), accounting systems being, sometimes, based on the national culture values the researches that had developed them. The Balanced Scorecard, accounting systems developed by american researches Kaplan and Norton, are being used in a lot of countries outside of United States of America, Brazil included. The analysis of the excellent cultural dimensions for the Balanced Scorecard's implementation results in some theorical proposal about Balanced Scorecard's implementation in brazilian companies, showing that culture values in Balanced Scorecard framework match within american culture values, and dismatch brazilian culture values. The results from the analisys from five case studies
\end{abstract}


A implantação do balanced scorecard em empresas brasileiras sob a perspectiva da cultura nacional

Frederico Pires Pereira Fernandes, Ana Carolina Pimentel Duarte da Fonseca

about Balanced Scorecard's implementation in brazilian companies show the influence of the cultural differences during the implantation process.

Key words: Balanced scorecard. Organizational Culture. National Culture.

\section{INTRODUÇÃO}

O Balanced Scorecard, modelo gerencial desenvolvido por Kaplan e Norton, tem entre os seus princípios transformar a estratégia em um processo contínuo e, através da utilização de medidas de desempenho, estabelecer uma relação de causa e efeito entre a estratégia e a operação. "O Balanced Scorecard traduz a missão e a estratégia das empresas num conjunto abrangente de medidas de desempenho que serve de base para um sistema de medição e gestão estratégica" (KAPLAN e NORTON, 1997, p. 2). As medidas propostas pelo Balanced Scorecard são compostas tanto por critérios objetivos quanto subjetivos, entre eles elementos comportamentais, como a motivação.

Dados de 2001 de uma pesquisa da consultoria Bain \& Co. já indicavam que $50 \%$ das empresas americanas listadas na Fortune 1000 haviam adotado o Balanced Scorecard e que, nos países da Europa, a taxa de adoção estava entre 40 e $45 \%$ (EMANAGER apud SOARES JR, 2003).

Diversos autores, entre eles Hofstede (1991), Hampden-Turner e Trompenaars (1995), e Caldas e Wood (1998) alertam que os sistemas gerenciais geralmente carregam características culturais de quem os desenvolveu. Ou seja, aspectos determinantes da cultura nacional daqueles que elaboraram práticas gerenciais interagem com os valores nacionais dos membros da organização que as utiliza, interferindo na sua implantação e aceitação.

Da mesma forma que a cultura, a cultura organizacional está em contínuo desenvolvimento, é algo socialmente produzido e reproduzido através do tempo, influenciando o comportamento das pessoas com relação ao uso da linguagem, tecnologia, regras, leis, conhecimento e idéias (MEEK, 1988). De acordo com Allaire e Firsiroto (1984), a cultura organizacional pode ser influenciada por três fatores que se combinam e competem entre si: os valores e características da sociedade em que a organização se encontra, a história da organização e lideranças passadas e fatores 
A implantação do balanced scorecard em empresas brasileiras sob a perspectiva da cultura nacional

Frederico Pires Pereira Fernandes, Ana Carolina Pimentel Duarte da Fonseca

contingenciais como tecnologia, características da indústria, etc. Assim, a adoção de práticas gerenciais desenvolvidas em outros países influencia a cultura organizacional pois nela introduz valores e características das sociedades onde foram desenvolvidas

Desta forma, é possível que, o Balanced Scorecard, modelo gerencial desenvolvido nos EUA, contenha em seus arcabouços teóricos elementos da cultura norte- americana, ou seja, valores distintos daqueles que permeiam as organizações brasileiras, e que, portanto, interagem com os presentes na cultura das organizações brasileiras.

Para compreender como se processam as influências da cultura nacional sobre a cultura organizacional, é importante destacar em que aspectos as nações podem diferir. Segundo Hofstede (1991), os antropólogos, desde o início do século XX, vêm chamando a atenção para o fato de que as sociedades têm que lidar com os mesmos problemas, mas respondem a eles de formas distintas. Esses problemas representam dimensões das culturas, ou seja, aspectos que ocorrem em combinação e que podem ser comparados entre culturas.

Através da observação de estudos de casos de implantação do Balanced Scorecard em empresas brasileiras, desenvolvidos por Soares Jr. (2003) e Pessanha (2004),buscou-se observar se as diferenças entre os valores da cultura brasileira e os contidos nesta prática gerencial influenciam sua implantação em empresas brasileiras. Para atingir este objetivo foram selecionadas algumas dimensões propostas por Hofstede (1991) e Trompenaars (1995) que se relacionam com os aspectos enfatizados no arcabouço teórico do Balanced Scorecard e que apresentam grande divergência nas posições do Brasil e Estados Unidos..

Nas próxima seção, apresenta-se uma síntese das principais características do Balanced Scorecard. Em seguida, é feita uma descrição sucinta das dimensões culturais selecionadas, bem como da posição das culturas norte-americana e brasileira nessas dimensões. Na seção seguinte, são destacados os valores culturais implícitos nas características do modelo e apresentadas algumas proposições teóricas decorrentes da interação da cultura brasileira com essas mesmas características. A seguir, examinam-se os processos de implantação do Balanced Scorecard em cinco 
A implantação do balanced scorecard em empresas brasileiras sob a perspectiva da cultura nacional

Frederico Pires Pereira Fernandes, Ana Carolina Pimentel Duarte da Fonseca

empresas brasileiras, procurando-se verificar se as implicações identificadas com base na teoria ocorreram na prática. Finalmente, são expostas as considerações finais.

\section{ARCABOUÇO TEÓRICO DO BALANCED SCORECARD}

Desenvolvido por Kaplan e Norton (1997) o Balanced Scorecard é apresentado como um sistema de informações capaz de agregar as informações financeiras e nãofinanceiras, propiciando aos gestores transmitir e executar a missão e a estratégia da empresa. Kaplan e Norton (1997) entendem que os atívos intangíveis tornaram-se decisivos para criação de valor sustentável para as organizações. Dentre os componentes dos ativos intangíveis, os autores citam o capital organizacional, definido como a capacidade da organização mobilizar e sustentar o processo de mudança necessário para executar a estratégia. O desenvolvimento do capital organizacional proporcionaria a compreensão comum da visão, dos valores e da estratégia, criando uma cultura voltada para o desempenho.

O Balanced Scorecard, conforme apresentado pelos autores, consiste em um sistema de objetivos e medidas, alinhados entre si, agrupados em quatro perspectivas, sendo elas:

a) Perspectiva financeira, que apresenta objetivos e indicadores relacionados à criação de valor para os sócios.

b) Perspectiva dos clientes, apresenta objetivos e indicadores relacionados à criação de valor para os clientes.

c) Perspectiva dos processos internos, que apresenta objetivos e indicadores relacionados à criação de valor para a empresa, do ponto de vista dos processos;

d) Perspectiva de aprendizado e crescimento, que apresenta os objetivos e indicadores relativos à criação de valor para os funcionários.

Kaplan e Norton (1997) defendem a integração entre o planejamento estratégico e o orçamento operacional para que a ação seja vinculada à visão da organização. A comunicação dos objetivos e medidas do Balanced Scorecard não é suficiente para 
A implantação do balanced scorecard em empresas brasileiras sob a perspectiva da cultura nacional

Frederico Pires Pereira Fernandes, Ana Carolina Pimentel Duarte da Fonseca

obter o comprometimento de todos os membros. Kaplan e Norton (op.cit.) entendem que o scorecard corporativo pode e deve ser traduzido em scorecards correspondentes para os níveis hierárquicos inferiores, proporcionando o alinhamento das metas de cima para baixo. Os autores recomendam que os planos de recompensas dos empregados estejam atrelados ao cumprimento das metas do Balanced Scorecard, sugerindo inclusive o estabelecimento de limites mínimos para os objetivos estratégicos, e a utilização de julgamentos subjetivos para a remuneração. Os autores entendem que a remuneração baseada somente em critérios objetivos pode criar desequilíbrios para a estratégia da organização, pois estes critérios incentivariam os funcionários a alcançarem seus objetivos pessoais, e a não se envolverem nos objetivos da organização.

Em suas pesquisas, Kaplan e Norton (2001)observaram um padrão consistente entre a utilização do Balanced Scorecard e o sucesso na administração da estratégia. Ressaltando diferenças de abordagem, seqüência e ritmo, os autores afirmam terem observado a atuação do que denominaram de "princípios da organização focalizada na estratégia", abaixo descritos:

Traduzir a estratégia em termos operacionais.

Os mapas estratégicos descrevem e comunicam a estratégia para a organização de maneira mais disciplinada, aumentando a probabilidade de sucesso da organização na implantação da estratégia, na medida em que criam um ponto de referência comum e compreensível para todas as unidades e empregados.

\section{Alinhar a organização à estratégia.}

As organizações são projetadas em torno de diversas especialidades, e cada uma função tem seu próprio corpo de conhecimentos, linguagem e objetivos. As organizações devem utilizar a estratégia para criar sinergias entre as unidades de negócios e os serviços compartilhados. Segundo os autores, o Balanced Scorecard permite a organização criar estas sinergias de maneira coordenada.

Transformar a estratégia em tarefas de todos. 


\section{A implantação do balanced scorecard em empresas brasileiras sob a perspectiva da cultura nacional}

Frederico Pires Pereira Fernandes, Ana Carolina Pimentel Duarte da Fonseca

As organizações focalizadas na estratégia usam o Balanced Scorecard em três processos diferentes para o alinhamento dos empregados com a estratégia. Em primeiro lugar, na comunicação e educação onde os empregados devem conhecer e compreender a estratégia a fim de que contribuam para sua implantação. Em segundo lugar, no desenvolvimento de objetivos pessoais e de equipes onde os empregados devem compreender de que maneira são capazes de influenciar a implantação bemsucedida da estratégia. Finalmente, nos sistemas de incentivos e recompensas onde os empregados devem sentir que compartilham as recompensas provenientes dos êxitos da organização, o que representa a conexão entre desempenho organizacional e recompensas individuais.

Como converter a estratégia em processo contínuo.

Uma das propostas do Balanced Scorecard é estabelecer uma aproximação do processo orçamentário, alicerce dos sistemas de gestão tradicionais, com o planejamento estratégico das organizações, equilibrando assim objetivos de curto e longo prazos. A proposta dos autores se baseia em três conceitos: conexão entre estratégia e orçamento; sistemas de feedback estratégico interligados ao Balanced Scorecard; e experimentação, aprendizado e adaptação.

Como mobilizar a mudança por meio da liderança executiva.

A experiência mostrou que é difícil transferir para a prática os preceitos do planejamento estratégico. Não só a transferência é difícil, como também as tentativas de instalar uma disciplina estratégica rigorosa provocaram uma resistência ao planejamento, inércia organizacional que parece frustar todos os esforços e rejeitar as tentativas de planejamento. Como forma de vencer esta resistência, enfatiza-se a necessidade de dedicação da alta administração. 
A implantação do balanced scorecard em empresas brasileiras sob a perspectiva da cultura nacional

Frederico Pires Pereira Fernandes, Ana Carolina Pimentel Duarte da Fonseca

\section{AS DIMENSÕES DA CULTURA NACIONAL E O BALANCED SCORECARD: PRINCIPAIS DIFERENÇAS ENTRE A CULTURA BRASILEIRA E A CULTURA AMERICANA}

Com base nas principais características do Balaced Scorecard e nas pesquisas de Hofstede(1991) e Trompenaars (1995), foram selecionadas algumas dimensões culturais que se julgou relevantes no processo de implantação do Balaced Scorecard em empresas brasileiras. As dimensões foram escolhidas por se relacionarem com aspectos inerentes ao Balanced Scorecard e por apresentarem grandes divergências nas posições do Brasil e dos Estados Unidos. Foram examinadas as seguintes dimensões: individualismo versus coletivismo, universalismo versus particularismo, culturas difusas versus culturas específicas, distância do poder e status pela conquista versus status atribuído. A seguir, são apresentadas as posições do Brasil e dos Estados Unidos em cada uma dessas dimensões e suas implicações na implantação do Balanced Scorecard em empresas brasileiras.

\section{Individualismo versus coletivismo}

Os traços culturais que tratam da relação do indivíduo com o grupo foram englobados por Hofstede em uma única dimensão: individualismo versus coletivismo . Já Trompennars (1995) desmembrou os aspectos estudados em três dimensões: individualismo versus coletivismo, universalismo versus particularismo e culturas difusas versus culturas específicas.

Hofstede (1991, p.51) considera individualistas as sociedades em que os laços dos indivíduos são frouxos. Espera-se que as pessoas cuidem de si e de seu núcleo familiar. Por outro lado, nas sociedades coletivistas, as pessoas encontram-se ligadas, desde seu nascimento, a grupos coesos, que as protegem em troca de inquestinável lealdade. Assim, enquanto nas sociedades individualistas, a auto-realização pessoal é a meta almejada, nas nações coletivistas, os indivíduos se definem como parte de um grupo e buscam a aprovação do grupo a que pertencem. Da mesma forma, para Trompenaars (1995), esta dimensão trata do conflito entre o interesse do grupo e o 
A implantação do balanced scorecard em empresas brasileiras sob a perspectiva da cultura nacional

Frederico Pires Pereira Fernandes, Ana Carolina Pimentel Duarte da Fonseca

interesse de cada indivíduo. Citando Parson, descreve o individualismo como "uma orientação primordial ao eu", e o coletivismo como "uma orientação primordial aos objetivos e metas comuns". Esclarece, entretanto, que uma nação coletivista não é aquela que cerceia o livre arbítrio de cada indivíduo, e sim, aquela em que o desejo individual dos componentes do grupo é de que suas ações se reflitam positivamente no grupo como um todo.

No ambiente organizacional, principalmente nos critérios relacionados à remuneração, premiação e promoção dos seus integrantes, ficam explícitas as características que diferenciam o individualismo do coletivismo. Nem sempre é bem visto, em ambientes predominantemente coletivistas, a premiação do desempenho individual. $O$ destaque individual será bem aceito se este for decorrente de benefício proporcionado à coletividade, ou se este não implicar em atritos com os colegas. Em ambiente predominantemente individualista, a recompensa pessoal é bem aceita e desejada. Aquele que se diferencia dos demais, pelo mérito, torna-se referência para seus pares, que também desejarão possuir uma posição de destaque. Existe a percepção de que o aperfeiçoamento das organizações, em geral, é resultado do sucesso de cada um.

A orientação para os objetivos individuais em detrimento dos desejos do grupo é considerado o aspecto mais marcante da cultura norte-americana. A figura do selfmademan, ou seja, daquele que realizou conquistas através de seu próprio desempenho é desejada e motivo de admiração e respeito pelos demais membros da sociedade.

Por outro lado, na cultura brasileira, a motivação baseada em conquistas pessoais tende a ser interpretada como lesiva ao grupo, e portanto, não é socialmente bem aceita. Barbosa (1999) observa que a igualdade, como valor, não possui o mesmo significado na sociedade brasileira e na norte-americana. Nos EUA, ela se apresenta como um direito e pode resultar em desigualdade, pois um melhor desempenho por parte do indivíduo ou de um grupo os intitula a uma posição diferenciada em relação aos demais. No Brasil, invoca-se também uma igualdade moral que tem origem na idéia de unidade biológica do ser humano. Neste sentido, a autora afirma que o Brasil rejeita a ideologia meritocrática em função da concepção de igualdade prevalecente entre nós. 


\section{A implantação do balanced scorecard em empresas brasileiras sob a perspectiva da cultura \\ nacional \\ Frederico Pires Pereira Fernandes, Ana Carolina Pimentel Duarte da Fonseca}

Nas palavras da autora:

O esforço de cada um, a vontade de realizar (achievement) e as diferenças de talento naturais não funcionam como vetores que transformam indivíduos comuns em winners, como na sociedade norte-americana, mas como expressões "objetivadas" de processos históricos e sociais sobre os quais os indivíduos têm pouco controle. Como os indivíduos nascem em posições sociais distintas, eles desenvolvem habilidades desiguais. Elas não são naturais, no sentido de inatas. Portanto, os resultados positivos e negativos advindos das habilidades e talentos de cada um não são responsabilidade do indivíduo, e sim de todo corpo social. Daí a lógica distributiva que subjaz à concepção de igualdade substantiva brasileira. (BARBOSA,1999, p. 65-66)

As práticas de compensação presentes na cultura brasileira indicam a preferência por compensações para fazer justiça social. De acordo com Barbosa (1999, p.71), o único valor legítimo na perspectiva igualitária radical é a senioridade, pois é "um atributo ao alcance de todos, porquanto só depende da simples permanência no emprego", enquanto o mérito, "depende do indivíduo e de suas especificidades, nem todos o possuem ou podem alcançá-lo"

Além dos aspectos acima mencionados, Hofstede inclui dentro desta dimensão outras características que Trompennars atribui separadamente a culturas universalistas e particularistas e a culturas difusas e específicas.

\section{Universalismo versus particularismo}

Conforme observa Trompennaars (1995) o universalismo é a tendência a estabelecer leis universais para os fenômenos. Ao estabelecer códigos pré-definidos ou padronizados, ele simplifica a realidade, ignorando particularidades. Também busca a eqüidade entre seus membros. As relações baseiam-se em contratos, regras e normas. Exceções não são bem aceitas e dificilmente serão amparadas e absorvidas pelos demais membros. A regulamentação das relações é necessária para transformar em real um conceito um tanto abstrato, "o bem comum". Por sua vez, o particularismo concentra-se na circunstância excepcional à vista dos presentes. Assim, o julgamento depende da relação entre os atores da situação. Quanto mais estreita for esta relação, maior a tendência de que esta não siga a regra formal.

Nos negócios, uma das implicações das diferenças nesta dimensão é a 
A implantação do balanced scorecard em empresas brasileiras sob a perspectiva da cultura nacional

Frederico Pires Pereira Fernandes, Ana Carolina Pimentel Duarte da Fonseca

importância dada aos contratos. Nas culturas universalistas, a forma de codificar o que foi acordado entre as partes é o contrato. Qualquer quebra no mesmo, será resolvida por advogados. Nas culturas particularistas, as relações pessoais são muito mais importantes do que os contratos, sendo que muitas vezes cláusulas preventivas podem ofender alguns participantes e impedir o desenvolvimento de boas relações de trabalho.

Da mesma forma, nas culturas particularistas, existe uma tendência a adaptar os cargos às pessoas, ao invés de buscar as pessoas mais adequadas à descrição dos cargos, como propõem as empresas que atuam em sociedades universalistas.

Com a globalização, as empresas universalistas, que buscam padronizar suas operações nos diversos países em que atuam, encontram resistência nas subsidiárias localizadas em culturas particularistas, que preferem, por sua vez, manter padrões locais. Muitas vezes, elas fingem se submeter às regras da matriz, adotando um comportamento ritualístico.

Os americanos apresentam uma tendência a fazer simplificações da realidade, estabelecendo códigos pré-definidos ou padronizados. A própria determinação dos Estados Unidos em proclamar a "supremacia" da Administração de Empresas, estabelecendo para si próprio a tarefa de fazer uma ciência da administração, produzindo uma grande quantidade técnicas e fórmulas com o objetivo de construir um código de administração universal, espelha esta característica. Da mesma forma, esta tendência universalista de seguir soluções pré-determinadas explica a dificuldade dos americanos em lidar com a participação e a preferência por decisões individuais. Fica difícil ter "boas relações humanas" se a relação é controlada por apenas uma das partes. As culturas universalistas têm dificuldade de permitir a participação dos funcionários na tomada de decisões pois acreditam que estas possam se basear em explicações universais. Como a participação demanda conexões particularistas, só é válida se o chefe se encontra preparado para mudar de idéia em função das sugestões recebidas, o que implica que a solução não deve seguir uma receita pré-determinada. (HAMPDEN-TURNER e TROMPENAARS,1995)

Prates e Barros (1997) colocam que a conjunção da concentração do poder e a existência das relações pessoais cultivadas pela figura do "padrinho" na cultura 
A implantação do balanced scorecard em empresas brasileiras sob a perspectiva da cultura nacional

Frederico Pires Pereira Fernandes, Ana Carolina Pimentel Duarte da Fonseca

brasileira, denominada paternalismo, gera dupla dependência e, consequentemente, um menor grau de liberdade e autonomia para seus membros, mas abre caminho para uma forma eficiente de se identificar e pertencer a um grupo, proporcionado um maior grau de segurança a seus membros. O paternalismo contêm elementos característicos do particularismo de Trompenaars, pois o indivíduo espera ter um reconhecimento diferente daquele que não está integrado ao grupo, ou seja, não espera que seja aplicado a ele a mesma regra que será aplicado aos indivíduos que não integram o grupo. Existe um desejo e uma aceitação tácita das normas e regras, mas com uma prática distorcida e reinterpretações ajustadas com o nosso desejo. "Se a pessoa é de nossas relações ou com autoridade, a flexibilidade na reinterpretação é ampla; se é pessoa de fora das relações, a rigidez é absoluta." (PRATES e BARROS, 1997, p. 64)

Assim, a preferência por decisões individuais não parece adequada à cultura brasileira, onde a ênfase nas relações pessoais convida à participação de todos na discussão dos assuntos. No entanto, a participação não envolve necessariamente atribuição de responsabilidade aos indivíduos, em função do alto grau de distância do poder, como será visto no próximo item.

Fonseca (1997), sintetizando alguns depoimentos de brasileiros sobre tomada de decisão dentro do sistema de planejamento e controle, aponta na mesma direção:

Os informantes declararam que gostam que o chefe peça a sua opinião e se sentem motivados com isso. Entretanto, quando os problemas tomam maiores proporções, os chefes são envolvidos na tomada de decisão pois os indivíduos preferem não fazer nada com medo de perder os seus empregos, evitando tomar a decisão. [...] Assim, de um modo geral, parece que no Brasil todos gostam de participar das decisões mas a tomada de decisão deve ser do chefe. (FONSECA, 1997, p. 237)

Segundo Hampden-Turner e Trompenaars (1995) um dos fatores que contribui para o sucesso econômico americano é o valor que é dado ao universalismo, que se refere à eqüidade entre seus membros. A eqüidade, geralmente conduzida por meio de regras e normas universais, busca garantir direitos e liberdade aos indivíduos. Beekun, Stedham e Yamamura (2003) corroboram a idéia de igualdade observando que os americanos consideram ético a aplicação equalitária de regras e normas para todas as 


\section{A implantação do balanced scorecard em empresas brasileiras sob a perspectiva da cultura nacional \\ Frederico Pires Pereira Fernandes, Ana Carolina Pimentel Duarte da Fonseca}

partes.

O pensamento americano de que todos devem ter oportunidades iguais, aliado a idéia de vencer, faz com que os negócios sejam encarados como um jogo, sendo o sucesso econômico o resultado de uma série de competições entre firmas, em que os números indicam mensalmente quem está vencendo. Por esta razão, as firmas britânicas e norte-americanas freqüentemente estabelecem "mercados internos" dentro das corporações em que os centros de lucro competem por recursos.

DaMatta (2000, p.25,) apresenta uma interpretação dualista da cultura brasileira, em que haveria um código da casa, centrado na família, na amizade, na lealdade, na pessoa e no compadrio, e um código da rua, fundamentado em leis universais, numa burocracia antiga e num formalismo jurídico legal excessivo. Entretanto, para o autor, o que possibilita uma interpretação correta da sociedade brasileira é a possibilidade de estudar o que está "entre as coisas". Sendo uma sociedade relacional, o valor fundamental é sintetizar modelos e posições, contrapondo-se a nações protestantes como os Estados Unidos. Nas palavras de DaMatta:

Assim, nos Estados Unidos há exclusão e separação; no Brasil, há junção e hierarquização. Num caso o credo diz: iguais, mas separados; noutro ele decreta: diferentes, mas juntos. Lá o indivíduo é o sujeito do sistema; aqui, o sujeito não é o indivíduo, mas também a relação, o elo, o ponto de ligação. (DAMATTA, 2000, p.108)

Assim, facilidade de lidar com a ambiguidade e sintetizar, descrita acima sugere uma capacidade de lidar com informações pouco precisas e de extrair do contexto aspectos não contemplados nas medidas quantitativas. Assim, na avaliação dos centros de lucro, a sociedade brasileira aceita que outros aspectos, além dos de natureza financeira, sejam levados em consideração.

\section{$\underline{\text { Culturas difusas versus culturas específicas }}$}

De acordo com Trompenaars (1995), as culturas específicas caracterizam-se pela imposição dos limites e barreiras entres os "espaços da vida". As relações se moldam conforme a situação específica. Por exemplo, gerente e subordinado manterão 


\section{A implantação do balanced scorecard em empresas brasileiras sob a perspectiva da cultura nacional \\ Frederico Pires Pereira Fernandes, Ana Carolina Pimentel Duarte da Fonseca}

todas as normas de hierarquia e status no trabalho, mesmo sendo, fora das delimitações do trabalho, amigos de longa data. A diferença no tratamento entre eles, nos diferentes "espaços da vida", não será vista como desrespeito ou traição por nenhum dos dois. Em culturas difusas, cada "espaço da vida" permeia todos os outros. A relação entre gerente e subordinado não está restrita às questões oriundas da empresa. Ela influencia as relações nos demais espaços.

Outra questão que diferencia estas culturas é o tamanho dos espaços públicos e privados. Nas culturas específicas, o espaço público é maior do que o privado e está dividido em várias seções. O fato de alguém ser admitido em uma delas, não significa que as outras tenham sido abertas. Nas culturas difusas, o espaço privado é maior e é muito mais difícil penetrá-lo, mas do momento em que se é admitido em alguma de suas partes, o acesso às demais é praticamente automático.

A questão básica desta dimensão é a perda do prestígio (perda da face), que acontece quando alguma coisa que as pessoas percebem como privada se torna pública. Nas culturas específicas, onde a área privada é claramente separada da vida pública, a confrontação de idéias e o discurso direto não são tratados como uma questão pessoal e, conseqüentemente, não provocam, de forma ampla, a perda de prestígio, nem interferem na privacidade de cada um. Nas culturas difusas, a exposição pública é vista com certo temor. Evitam-se as confrontações diretas, danosas aos participantes, já que tudo é tratado como pessoal .

Culturas específicas valorizam a meritocracia baseada no desempenho e no cumprimento de metas, tendo no ambiente de negócio uma relação clara entre o objetivo observado e as conseqüências pelo cumprimento, ou não, das premissas estabelecidas. Já nas culturas difusas, existe a idéia de que as relações são mais importantes do que os resultados, sendo impossível atribuir a responsabilidade a apenas uma pessoa pois o resultado por ela alcançado pode ser fruto do esforço de outros. Valoriza-se a lealdade entre seus membros, o que acarreta uma menor rotatividade de empregados e a adoção de critérios de remuneração e avaliação mais subjetivos .

A cultura americana possui um espaço público muito maior do que o privado. 


\section{A implantação do balanced scorecard em empresas brasileiras sob a perspectiva da cultura \\ nacional}

Frederico Pires Pereira Fernandes, Ana Carolina Pimentel Duarte da Fonseca

Por exemplo, como os americanos se mudam com muita facilidade, carros, mobílias podem ser considerados bens semi- públicos, ao contráro de outras culturas difusas, onde esses bens pertencem ao espaços privado. Além disso, o espaço público dos americanos é segmentado em várias seções específicas. Quem penetra em algum desses espaços, não deve se sentir íntimo, pois não significa que conseguirá penetrar nos demais.

A busca pela compreensão dos aspectos culturais da sociedade brasileira remete a uma análise do processo de colonização ocorrido no país. DaMatta (1997) cita a relação do senhor de engenho e escravos, onde o primeiro, era não só considerado dono do trabalho do escravo, mas até mesmo responsável por sua moral. Até hoje, se mistura a relação puramente econômica com laços pessoais de simpatia e amizade. Prates e Barros (1997) também indicam uma estrutura familiar coesa e quase inflexível, centralizada no poder patriarcal, no estabelecimento dos padrões morais dos seus membros, onde prevalecem as preferências por laços afetivos. Christensen e Rocha (1986) complementam que a autoridade está baseada mais nas relações pessoais do que na estrutura hierárquica. A afirmação dos autores denota uma característica relacionada a culturas difusas, em que não há separação entre os diversos espaços da vida, e portanto, do momento que alguém é admitido em um deles, acaba entrando em todos os outros.

Os americanos, sendo culturas específicas, se sentem motivados com a administração por objetivos e aceitam a avaliação e remuneração com base na sua performance, ou seja, no alcance de objetivos específicos. Também não se importam com o discurso direto, pois sabem separar seu espaço privado do público, não levando para o lado pessoal, eventuais críticas (TROMPENAARS. 1995). Conforme ressalta Lewis (1996, p.79), eles "precisam de feedback constante, encorajamento e elogios do seu executivo sênior". Entretanto, para Schneider (1988), o ato de dar feedback direto não leva em conta a idéia de harmonia (saving face), tão crucial nas culturas difusas, onde confrontar um empregado discutindo fracasso de uma maneira direta e aberta pode ser muito constrangedor.

Segundo Oliveira e Machado-da-Silva (2001) o brasileiro espera do seu local de 
A implantação do balanced scorecard em empresas brasileiras sob a perspectiva da cultura nacional

Frederico Pires Pereira Fernandes, Ana Carolina Pimentel Duarte da Fonseca

trabalho hospitalidade e harmonia, e não luta e competição. Espera se sentir em casa, e que a organização reproduza a família. Como as relações pessoais misturam-se com as de trabalho, qualquer crítica ao trabalho é levada para o campo pessoal. Assim, as organizações brasileiras se caracterizam por uma interação social intensa entre seus membros, onde se busca a harmonia e o conflito não é desejável, principalmente, como destaca Tanure (2005, p.107), pelos subordinados. Ilustra bem este fato a declaração feita por um funcionário da subsidiária de uma multinacional, em pesquisa realizada por Fonseca (1997), que observou que o feedback tende a ser "construtivo para a empresa, mas destrutivo para a minha pessoa".

\section{Distância do poder}

Segundo Hofstede (1991), considerando que existe deigualdade em qualquer sociedade, a dimensão distância de poder mede o grau de aceitação da diferença de poder entre os membros de um grupo. A distância de poder se refere à medida em que os participantes menos poderosos das instituições e organizações dentro de um país aceitam a distribuição desigual de poder.

Em países identificados com alto grau de distância do poder, os empregados frequentemente demonstram medo em não concordar com seus superiores, e também revelam preferir chefes autocráticos ou paternalistas do que chefes consultivos. Isto revela uma considerável dependência dos empregados para com seus superiores. Nestes países, gerentes e subordinados não se colocam em patamar de igualdade, e o sistema hierárquico se baseia na desigualdade existente, e por assim dizer, coloca "cada um no seu devido lugar". Hofstede (1991) relata algumas características encontradas no ambiente organizacional de alta distância do poder tais como: estruturas hierárquicas verticalizadas, poder concentrado nas mãos de poucas pessoas, privilégios para os níveis hierárquicos mais altos, chefes autocráticos e paternalistas.

Em países caracterizados pela baixa distância de poder, superiores e subordinados consideram um ao outro essencialmente como iguais, e o sistema hierárquico das organizações, reflete uma desigualdade que é estabelecida só por 
A implantação do balanced scorecard em empresas brasileiras sob a perspectiva da cultura nacional

Frederico Pires Pereira Fernandes, Ana Carolina Pimentel Duarte da Fonseca

conveniência, pois o subordinado de hoje pode ser o superior de amanhã . Tanto superiores quanto subordinados preferem um estilo consultivo a um estilo autoritário.

A sociedade americana caracteriza-se por uma baixa distância do poder. As relações de poder são vistas como momentâneas, determinadas pelos papéis de cada um dentro da estrutura organizacional. Os chefes costumam ser acessíveis para os subordinados.

De uma maneira geral, a sociedade brasileira pode ser caracterizada como uma sociedade hierárquica em que as relações pessoais são muito importantes, repetindo a situação existente na época dos senhores de engenho, onde, conforme observa Motta (1997, p.:31), "a distância social era contrapartida da proximidade física."

As organizações brasileiras costumam apresentar alto grau de autoritarismo, com imposições dos níveis hierárquicos mais altos para níveis inferiores, que refletem os desejos e crenças dos líderes. Ao seguir as normas do plano formal, ou seja as declarações explícitas dos desejos e crenças dos superiores, os subordinados entendem que estão contribuindo para a harmonia da organização e esperam ser recompensados, por seus superiores, por se manterem leais as suas determinações.

Este comportamento tem como contrapartida o mutismo e a baixa consciência crítica, tendo como consequência baixa iniciativa, pouca capacidade de realização por autodeterminação e transferência de responsabilidade das dificuldades para as lideranças. Este traço cultural, denominado de "postura de espectador.", geralmente é identificado em países com alto índice de distância de poder. (PRATES e BARROS, 1997)

A postura de espectador dificulta a atribuição de responsabilidade aos indivíduos nas organizações brasileiras. Reforçando este traço, destaca-se o medo de errar que é, segundo Tanure (2005, p.108), "a contraparte do paternalismo" que, por sua vez, levaria ao "desejo de acertar sempre e assim ser reconhecido pelo grande poder".

Para Tanure (2005), a flexibilidade é uma característica fundamental do sistema de ação cultural brasileiro. Ela pode ser desmembrada em adaptabilidade e criatividade. A primeira refere-se à capacidade que as empresas demonstram em se ajustarem a mudanças de regras. A adaptabilidade não produz nada de novo. Já a 
A implantação do balanced scorecard em empresas brasileiras sob a perspectiva da cultura nacional

Frederico Pires Pereira Fernandes, Ana Carolina Pimentel Duarte da Fonseca

criatividade possui um elemento inovador. Entretanto, em função da postura de espectador e do medo de errar, a criatividade só se manifesta em situações de igualdade, tanto de fato como de direito, como pode ser observado no futebol ou no carnaval, onde ocorre a expressão da singularidade, do potencial de cada um.

\section{Status pela conquista e status atribuído}

Para Trompenaars (1995), o status pela conquista é concedido pela sociedade a seus membros de acordo com as atividades que estes exercem, enquanto que, o status atribuído, é conferido às pessoas em função de sua origem, sexo, idade, instrução, ou seja, por fatores não relacionados ao desempenho.

Para Schneider (1988, p.11), quando a avaliação é feita com base na realização, admite-se que "a performance, isto é, o que foi feito ou realizado é importante e pode ser medido objetivamente. O que é avaliado é então, comportamento e, não, características". Por outro lado, acrescenta a autora que, quando o que conta é o status atribuído, como ocorre nas firmas japonesas, "existe uma maior preocupação em julgar a integridade, a conduta moral, a lealdade e o espírito cooperativo de uma pessoa do que em alcançar um alto volume de vendas".

Os resultados da pesquisa de Trompenaars (1995) identificaram alta correlação entre a orientação religiosa e a forma de concessão do status. As sociedades predominantemente católicas, budistas e hinduístas tendem a orientar o status pela atribuição, e as sociedades predominantemente protestantes tendem a orientar o status pela conquista.

Os americanos enfatizam a conquista do status através do desempenho individual. A avaliação na cultura americana tem por base o desempenho do empregado, ou seja, o status alcançado, um forte traço cultural da formação dos Estados Unidos. Por outro lado, a avaliação com base no desempenho, a meritocracia, não tem sido a forma mais usada nas empresas brasileiras pois não atende à nossa concepção de igualdade moral e nem ao sistema de relações pessoais 
A implantação do balanced scorecard em empresas brasileiras sob a perspectiva da cultura nacional

Frederico Pires Pereira Fernandes, Ana Carolina Pimentel Duarte da Fonseca

Assim, o que se constata é, primeiro, uma rede de significados em que sistemas meritocráticos possuem forte ligação com os sistemas de seleção, mas não com sistemas de avaliação. (Meritocracia do critério de limite mínimo) Há grande resistência cultural à avaliação enquanto conceito- principalmente quando se trata de estabelecer hierarquias dentro de grupos já formados, ou seja, à meritocracia baseada em critérios discriminatórios. Segundo, uma triangulação entre princípios meritocráticos, senioridade e relações pessoais está no centro dessa rede e a esgarça em diferentes direções conforme os contextos, servindo sempre como mecanismo de deslegitimação do mérito. (BARBOSA,1999, p.6364).

\section{IMPLICAÇÕES DAS DIFERENÇAS CULTURAIS NA IMPLANTAÇÃO DO BALANCED SCORECARD EM EMPRESAS BRASILEIRAS}

Examinado-se o arcabouço teórico do Balanced Scorecard sob uma perspectiva cultural, percebe-se uma correspondência entre as dimensões estudadas e algumas proposições de Kaplan e Norton (1997). Os valores culturais implícitos no Balanced Scorecard parecem coincidir com os da cultura americana e divergir dos da cultura brasileira nas seguintes dimensões.

- Individualismo: favorece o alinhamento dos objetivos individuais com os objetivos da organização, em que são valorizadas as conquistas e realizações pessoais.

- Universalismo: estimula a construção de uma estrutura de causa e efeito a partir de critérios objetivos (na maioria das vezes quantitativos) que vinculam as metas ao plano de remuneração

- Culturas específicas: aceitação de feedback na medida em que as críticas não são levadas para o lado pessoal

- Baixo grau de distância do poder: favorece a participação dos funcionários na definição dos objetivos e medidas de desempenho no processo de formulação e comunicação da estratégia, bem como no de discussão do feedback.

- Status pela conquista: aceitação de planos de remuneração baseados em medidas de desempenho 
A implantação do balanced scorecard em empresas brasileiras sob a perspectiva da cultura
nacional Frederico Pires Pereira Fernandes, Ana Carolina Pimentel Duarte da Fonseca

Apenas a orientação particularista da cultura brasileira pode favorecer a implantação do Balanced Scorecard pois permite uma maior aceitação de soluções não determinadas previamente e de critérios subjetivos no estabelecimento de planos de remuneração ligados às metas da organização.

Confrontando os elementos culturais americanos presentes no Balanced Scorecard com os elementos da cultura brasileira presentes nas dimensões culturais estudadas, foi possível identificar algumas proposições que retratassem possíveis obstáculos de origem cultural na implantação do Balanced Scorecard nas empresas brasileiras selecionadas.

- Proposição 1 - As empresas brasileiras preferem estabelecer objetivos voltados para grupos e equipes, dando pouca ênfase a conquistas e realizações pessoais.

- Proposição 2 - As empresas brasileiras apresentam dificuldade em aplicar regras universais a todos e em lidar com medidas quantitativas (critérios objetivos)

- Proposição 3 - As empresas brasileiras evitam a utilização de críticas no processo de comunicação de feedback para não ferir a harmonia do grupo

- Proposição 4 - O alto grau de distância do poder dificulta a participação dos funcionários no processo de formulação da estratégia pois existe, nas organizações brasileiras, por parte dos funcionários, a "postura de espectador" que prejudica o comportamento pró-ativo e inovador.

- Proposição 5 - A ênfase nas relações pessoais e no status atribuído dificultam a avaliação com base no desempenho

Por outro lado, também foi detectado um aspecto cultural que se mostra favorável à implantação do Balanced Scorecard em empresas brasileiras, descrito na proposição abaixo:

- Proposição 6: As empresas brasileiras levam em consideração conexões particularistas na tomada de decisão e aceitam critérios subjetivos no estabelecimento de planos de remuneração ligados às metas da organização. 
A implantação do balanced scorecard em empresas brasileiras sob a perspectiva da cultura nacional

Frederico Pires Pereira Fernandes, Ana Carolina Pimentel Duarte da Fonseca

\section{ANÁLISE DOS CASOS}

Foram examinados os processos de implantação do Balanced Scorecard em cinco empresas, a partir da análise dos estudos de caso de Soares Jr.(2003) e Pessanha (2004), buscando-se observar se as implicações culturais identificadas com base na teoria ocorreram na prática.

Soares Jr. (2003) analisou a experiência de implantação do Balanced Scorecard em onze empresas brasileiras, procurando identificar as principais dificuldades e ganhos obervados, bem como as soluções desenvolvidas e sua avaliação custobenefício. Nove casos tiveram como fonte de pesquisa os depoimentos apresentados durante o I Fórum Balanced Scorecard Brasil. Os outros dois casos foram investigados através de entrevistas realizadas pelo autor em uma subsidiária de uma empresa alemã que produz equipamentos eletrônicos e uma indústria química brasileira, sendo apenas estes últimos analisados na presente pesquisa.

Pessanha (2004) verificou a implantação do Balanced Scorecard em três empresas brasileiras - uma de telefonia celular espanhola, uma seguradora brasileira e um Banco de varejo controlado pelo governo federal brasileiro - e confrontou essas experiências práticas tanto com as propostas originais de Kaplan e Norton (1997;2001) como com as críticas direcionadas ao Balanced Scorecard encontradas na literatura.

A seguir, para cada proposição estabelecida na seção anterior será confrontado o comportamento ocorrido nas empresas com o que seria esperado com base na literatura.

\section{Proposição1}

A empresa de equipamentos eletrônicos relatou que o Balanced Scorecard ajudou a definir mais claramente os papéis e responsabilidades de cada setor e das pessoas envolvidas, porém a aplicação do Balanced Scorecard para o nível de objetivos individuais dependia de maiores investimentos e recursos em sistemas de tecnologia da informação.

A indústria química indicou que estava utilizando o Balanced Scorecard para o 
A implantação do balanced scorecard em empresas brasileiras sob a perspectiva da cultura nacional

Frederico Pires Pereira Fernandes, Ana Carolina Pimentel Duarte da Fonseca

alinhamento da estratégia e objetivos das unidades de negócios, e que estas informações eram utilizadas para a avaliação e desenvolvimento das competências dos funcionários em outras metodologias.

O estabelecimento de metas e objetivos do Balanced Scorecard na empresa de telefonia ficou restrito apenas aos gerentes e diretores de cada divisão, que definiram iniciativas e metas para suas equipes e seus participantes.

A seguradora e o banco de varejo não trataram de conquistas e realizações pessoais no desenvolvimento de seu Balanced Scorecard. Ambas empresas abordaram somente os objetivos das suas divisões ou unidades de negócios na implantação do sistema.

Como era esperado, em geral, identificou-se a preservação do comportamento coletivista, em que a organização trata dos objetivos e recompensas de grupos, divisões e diretorias, mas não os estabelece em nível individual. As empresas mantiveram os objetivos e suas medidas vinculados às equipes, preservando as relações com o grupo, porém estreitando os resultados de cada equipe com os resultados da organização. Somente uma das empresas manifestou a intenção de realizar o alinhamento dos objetivos pessoais com os objetivos da organização, e a sua vinculação aos planos de recompensas atrelados aos resultados apontados pelo Balanced Scorecard.

\section{Proposição 2}

Embora, de uma maneira geral, as empresas tenham afirmado que a implantação do Balanced Scorecard proporcionou-Ihes a capacidade de tornar o planejamento estratégico uma tarefa mais objetiva, duas delas fizeram ressalvas quanto à metodologia baseada na relação causa e efeito entre os objetivos e as medidas de desempenho, que resultaria numa simplificação da realidade. Na empresa de telefonia, o entrevistado disse que gostaria de melhorar as inter-relações entre os objetivos estratégicos, pois a firma ainda não está confortável com os relacionamentos de causa e efeito que foram traçados. No banco de varejo, as relações de causa e efeito foram estabelecidas de forma intuitiva, com base na experiência e na conversa, sem 
A implantação do balanced scorecard em empresas brasileiras sob a perspectiva da cultura nacional

Frederico Pires Pereira Fernandes, Ana Carolina Pimentel Duarte da Fonseca

relacionamentos unidirecionais. Novamente, este comportamento é típico de culturas particularistas que, conforme apresentado na discussão teórica, não se enquadram com facilidade em relações universais que simplificam a realidade.

Por outro lado, opondo-se às características particularistas, os entrevistados da seguradora e do banco de varejo concordam com o fato de que a alta administração presta relativamente pouca atenção às medidas específicas de cada unidade de negócio ou segmento, detendo-se mais nas medidas comuns (universais). Diferentemente do que era esperado, as organizações brasileiras mostraram-se familiarizadas com medidas financeiras de curto prazo, apenas indicando necessidade de outras medidas na definição das perspectivas para atender às necessidades das empresas. Pensou-se em adicionar uma perspectiva governamental na seguradora e uma perspectiva voltada para a sociedade no banco de varejo.

\section{Proposição 3}

A comunicação de feedback não foi observada, pois nenhuma das empresas havia implantado esta parte do processo. Ainda assim, as empresas observadas podem ser agrupadas em três conjuntos: as que entendiam que a aplicação da comunicação de feedback era importante; as que tratavam a comunicação de feedback como complemento do Balanced Scorecard; e as empresas que acreditavam que a comunicação de feedback não influiria nos ganhos propostos pelo Balanced Scorecard. Verifica-se, portanto, que nem todas as empresas estavam convencidas da importância do feedback.

No primeiro grupo está a empresa de equipamentos eletrônicos que declarou que a comunicação de feedback contribuiria para o aumento da sinergia dos membros da organização na formulação da estratégia e definição dos objetivos e metas, tendo em vista a maior participação dos funcionários no processo. No segundo grupo, a indústria química e a empresa de telefônia argumentaram que a comunicação de feedback seria uma conseqüência da própria aplicação do Balanced Scorecard, que deveria substituir ou ser aplicado simultaneamente com ferramentas e processos já utilizados pelas empresas. A seguradora e o banco de varejo formam o terceiro grupo. 
A implantação do balanced scorecard em empresas brasileiras sob a perspectiva da cultura
nacional Frederico Pires Pereira Fernandes, Ana Carolina Pimentel Duarte da Fonseca

Ambas empresas argumentam que os processos utilizados, até o momento, são eficientes e atendem às necessidades dos membros da organização.

\section{Proposição 4}

As observações sobre as relações com a hierarquia, que tratavam da participação dos funcionários no processo de formulação da estratégia, e a utilização do sistema de feedback, indicam que as empresas mantiveram um alto grau de distância do poder. Neste ponto é importante ressaltar que segundo os críticos de Kaplan e Norton, entre eles Malina e Selton e Simons (apud PESSANHA, 2004), o sistema de feedback do Balanced Scorecard favorece uma modelo de comunicação one-way, topdown e não participativa. Estas críticas favorecem a preservação de ambientes com alto grau de distância do poder. Somente a empresa de equipamentos eletrônicos relatou a ocorrência de feedback no sentido inverso, ou seja, down-top, devido a maior transparência sobre a estratégia da empresa e o estímulo à participação dos funcionários neste processo. Esta mesma empresa também observou a ocorrência de um estímulo ao processo inovativo e comportamento pró-ativo dos funcionários, confirmado a vinculação da criatividade a situações de igualdade, conforme sugerido na literatura.

\section{Proposição 5}

As empresas apresentaram um comportamento relacionado a culturas com status atribuído pois a adoção da metodologia do Balanced Scorecad não implicou necessariamente na ligação das metas estabelecidas no mesmo com seus planos de remuneração. Foram observados critérios subjetivos na avaliação de desempenho. Em três empresas não houve a ligação entre as metas estabelecidas no Balanced Scorecard e os planos de remuneração. No banco de varejo e na empresa de telefonia, esta ligação aconteceu de forma indireta, enquanto, na seguradora, foram usadas perguntas subjetivas dirigidas ao superior imediato ou contratos personalizados para avaliação de desempenho. 
A implantação do balanced scorecard em empresas brasileiras sob a perspectiva da cultura nacional

Frederico Pires Pereira Fernandes, Ana Carolina Pimentel Duarte da Fonseca

\section{Proposição 6}

A avaliação do desempenho e os critérios de recompensas pessoais foram estabelecidos, em grande parte, entre o funcionário e seu superior imediato, sendo esta uma atitude relacionada às culturas particularistas, ou seja, cada caso é analisado individualmente, preservando as situações específicas de cada um.

\section{CONSIDERAÇÕES FINAIS}

Butler et. al. (apud PESSANHA, 2004), em um estudo de caso de uma empresa inglesa, já haviam identificado problemas de incompatibilidade entre a estrutura do Balanced Scorecard e os valores culturais aceitos pelos funcionários, tendo como consequência o não reconhecimento da missão através Balanced Scorecard. Epstein e Manzoni (1998) acreditam que um dos obstáculos ao Balanced Scorecard pode ser a resistência de funcionários que não crêem no sucesso de sua implantação, nem nos objetivos traçados.

De uma maneira geral, as implicações culturais observadas na literatura foram observadas nas empresas estudadas, excetuando-se a aceitação de medidas quantitativas, o que pode ter ocorrido em função da possibilidade de utilização de critérios de recompensa que incorporavam conexões particularistas. Como foi observado na revisão teórica, existe espaço na cultura brasileira para outras medidas qualitativas, em função da ambiguidade, da ênfase nos relacionamentos e do próprio conceito de igualdade, que contradiz a idéia de meritocracia.

A alta distância hierárquica e a necessidade de manter a harmonia presentes na cultura brasileira parecem impedir que os funcionários digam o que pensam, minando iniciativas e repostas criativas, principalmente por parte dos funcionários que ocupam níveis inferiores da escala hierárquica. Da mesma forma, a utilização de feedback para corrigir erros pode ser prejudicada. Talvez não tenha sido por por acaso que, justamente na empresa em que ocorreu a participação dos funcionários, observou-se um comportamento inovativo e pró-ativo por parte dos funcionários.

Os resultados indicam, portanto, que é imprescindível que os executivos das 
A implantação do balanced scorecard em empresas brasileiras sob a perspectiva da cultura nacional

Frederico Pires Pereira Fernandes, Ana Carolina Pimentel Duarte da Fonseca

empresas liderem o processo de implantação do Balanced Scorecard, buscando identificar e compreender os elementos caracteríscos de sua própria cultura organizacional, de modo a analisar compatibilidade da mesma com os valores implícitos nesta ferramenta gerencial.

Vale ressaltar na análise das proposições a grande similaridade no comportamento e nas expectativas apresentadas pelas empresas que não são controladas por empresas estrangeiras quando comparadas com as análises obtidas nas demais empresas, principalmente na subsidiária da alemã, o que pode ser explicado pela influência da liderança dos executivos da empresa na condução do processo de implantação do Balanced Scorecard.

Não há neste trabalho a pretensão de proporcionar respostas definitivas sobre o tema, mas apenas de captar índicios sobre a questão cultural nas implantações do Balanced Scorecard em empresas brasileiras. Cabe ressaltar, entretanto que, apesar de terem sido analisados resultados alcançados em pesquisas que não tiveram como objetivo investigar aspectos culturais, o estudo sugere que estes parecem ter tido influência durante o processo de implantação, o que evidencia a importância de considerar as diferenças culturais no mesmo.

\section{REFERÊNCIAS}

ALLAIRE, Y.; FIRSIROTU, M. E. Theories of Organizational Culture. Organization Studies, v.5, n.3, p.193-226, 1984.

BARBOSA, L. Igualdade e Meritocracia: a ética do desempenho nas sociedades modernas. Rio de Janeiro: Editora Fundação Getúlio Vargas, 1999.

BEEKUN, R.I.; STEDHAM Y.; YAMAMURA, J. H. Business ethics in Brazil and the U.S.:A comparative investigation. Journal of Business Ethics; Feb 2003.

CALDAS, M.; WOOD, T. Antropofagia organizacional. In: ENCONTRO ANNUAL DA ASSOCIAÇÃO NACIONAL DOS PROGRAMAS DE PÓS-GRADUAÇÃO EM ADMINISTRAÇÃO, 22, 1998, Foz do Iguaçu. Anais Eletrônicos...Foz do Iguaçu: ANPAD, 1998. 1CD.

DAMATTA, Roberto. A casa \& a rua: espaço, cidadania, mulher e morte no Brasil. 6 ed. Rio de Janeiro: Rocco, 2000. 
A implantação do balanced scorecard em empresas brasileiras sob a perspectiva da cultura nacional

Frederico Pires Pereira Fernandes, Ana Carolina Pimentel Duarte da Fonseca

EPSTEIN, M., e MANZONI, J. Implementing corporate strategy: from tableux de bord to balanced scorecard. European Management Journal, v.16, n.2, pp 190-203, 1998.

FONSECA, A. C. P. D. Percepções de incerteza em um sistema de planejamento e controle: um estudo etnográfico Brasil-Inglaterra. Tese (Doutorado em Administração) Instituto COPPEAD de Administração Rio de Janeiro: Universidade Federal do Rio de Janeiro, 1997.

FREITAS, A. B. Traços Brasileiros para uma análise organizacional. In: PRESTES MOTTA, F. P.; CALDAS, M.P. (Orgs). Cultura organizacional e cultura brasileira. São Paulo: Atlas: 1997.

HAMPDEN-HAMPDEN-TURNER, C.; TROMPENAARS, F. The seven cultures of capitalism: value system for creatin wealth in the United States, Britain, Japan, Germany, France, Sweden and the Netherlands. London: Piatkus. 1994.

HOFSTEDE, G. Cultures and organizations: the software of the mind. London: McGraw Hill, 1991.

KAPLAN, R.S.e NORTON, D. P. A estratégia em ação: balanced scorecard. Rio de Janeiro: Campus, 1997.

Janeiro: Campus, 2001.

Organização orientada para a estratégia. Rio de

LEWIS, R. D. When cultures collides: managing successfully across cultures. London, Nicholas Brealey Publishing, 1996.

MEEK, V.L. Organizational Culture: Origins and Weaknesses. Organizational Studies, v.9, n.4, p.453-473, 1988.

MOTTA, F. C. P. Cultura e Organizações no Brasil. In:_e CALDAS, M.P. (Orgs). Cultura organizacional e cultura brasileira. São Paulo: Atlas: 1997.

OLIVEIRA, P.T.; MACHADO-DA-SILVA, C. L. Organizational Life Cycles and National Culture Traits: a study in the food industry in South Brazil. Anais Eletrônicos. BALAS 2001. 1CD.

PESSANHA, D. S. S. Obstáculos à implantação do Balanced Scorecard: estudos de casos em empresas brasileiras. 2004. Dissertação (Mestrado em Administração)-FACC, Universidade Federal do Rio de Janeiro, Rio de Janeiro, 2004.

PRATES, M. A.; BARROS, B. T. Os estilo brasileiro de administrar: sumário de um modelo de ação cultural brasileiro com base na gestão empresarial. In: PRESTES MOTTA, F.; CALDAS, M.P. (Orgs). Cultura organizacional e cultura brasileira. São 
Paulo: Atlas:1997.

PRESTES MOTTA, F. C. Cultura e organizações no Brasil. In: PRESTES MOTTA, F.; CALDAS, M. P. Cultura organizacional e cultura brasileira. São Paulo : Atlas, p. 25-37, 1997.

SCHNEIDER, S.C. National vs. corporate culture: implications for human resources management. INSEAD Working Papers Series, n.88/4. Fontainebleau: INSEAD, 1988.

SOARES JR., H. Experiências de implantação do Balanced Scorecard: como as empresas estão implantando o BSC no Brasil. 2003. Dissertação (Mestrado em Administração)-FACC, Universidade Federal do Rio de Janeiro, Rio de Janeiro, 2003.

TANURE, B. Gestão à brasileira: uma comparação entre América Latina, Estados Unidos, Europa e Ásia. São Paulo: Atlas, 2005.

TROMPENAARS, F. Nas ondas da cultura: como entender a diversidade cultural nos negócios. São Paulo: Educator, 1995. 